\title{
FEASIBILITY STUDY OF A COMBINED NEUTRON CENTRE FOR EUROPEAN RESEARCH AND TECHNOLOGY
}

\author{
A. Mosnier and E. Klein for the CONCERT team, CEA-DAPNIA Saclay, France
}

\section{Abstract}

The CONCERT concept started from a simple observation that a long series of applications could soon benefit from the outstandipower proton accelerators (HPPAs). These new linear accelerators should produce beams of several tens of MW. They could act as proton factories, with the protons being used to create large fluxes of other particles of interest : neutrons, other hadrons such as pions, muons, neutrinos and exotic ions. In comparison with existing proton drivers $(<1 \mathrm{MW})$, the expected increase in incident beam power will be reflected accordingly in the fluxes of secondary beams. For this reason, the latter are of the utmost interest for a series of applications :

- Condensed matter studies by neutron scattering

- Nuclear physics - study of Rare Ion Beams (RIBs)

- Accelerator Driven Transmutation (ADT) of longlived radioactive waste

- Technological Irradiation Tool (TIT) testing of materials

- Particle physics (muon colliders and neutrino factories)

Previous studies have shown that there is a broad overlap between these different applications in terms of proton beam specifications, so that one can think of combining a number of these applications on the same site with a single accelerator. One can also exploit other synergies on targets, conventional facilities, technical supports, computing, personnel, etc. The feasibility study in progress aims at exploiting this concept. The presentation will detail the status of the project.

\section{HPPA DRIVER SPECIFICATIONS}

Table 1 summarizes the beam requirements collected from all potential user communities in Europe. For condensed matter, the ESS [1] reference consists of $5 \mathrm{MW}$ short pulses at $50 \mathrm{~Hz}$ extracted from compressor rings and an additional $5 \mathrm{MW}$ long pulse option at $16.7 \mathrm{~Hz}$ is being contemplated. For the Rare Ion Beams, the EURISOL [2] project is based on the ISOL method and requires $200 \mathrm{~kW}$ power to create proton rich RIBs and even $5 \mathrm{MW}$ if spallation neutrons are used to create neutron-rich RIBs. For Accelerator Driven Transmutation, a minimum energy of $600 \mathrm{MeV}$ and a current of about $10 \mathrm{~mA}$ are required for a $100 \mathrm{MWth}$ demonstrator [3]. Though the original concept of an irradiation tool making use of spallation neutrons was based on a $40 \mathrm{MW}$ proton beam (SPALLAX project [4]), a lower power version of about $10 \mathrm{MW}$ is under consideration. Lastly, muon colliders and neutrino factories for particle physics demand significant R\&D; a preliminary design of a $2 \mathrm{GeV}$ superconducting driver was recently completed at CERN [5].

Table 1: Typical parameters for applications

\begin{tabular}{|c|c|c|c|}
\hline User & Beam Power & Energy & Current \\
\hline Condensed matter & $5 \mathrm{MW}$ & $1.3 \mathrm{GeV}$ & $3.75 \mathrm{~mA}$ \\
\hline $\begin{array}{c}\text { Radioactive Ions } \\
\text { from protons } \\
\text { from neutrons }\end{array}$ & $\begin{array}{c}\sim 200 \mathrm{~kW} \\
\sim 5 \mathrm{MW}\end{array}$ & $\begin{array}{c}>200 \mathrm{MeV} \\
\sim 1 \mathrm{GeV}\end{array}$ & $\begin{array}{c}\sim 1 \mathrm{~mA} \\
\sim 5 \mathrm{~mA}\end{array}$ \\
\hline Transmutation & & & \\
100 MWth demo & $\sim 5 \mathrm{MW}$ & $\sim 600 \mathrm{MeV}$ & $\sim 10 \mathrm{~mA}$ \\
Industrial system & $\sim 50 \mathrm{MW}$ & $\sim 1 \mathrm{GeV}$ & $\sim 50 \mathrm{~mA}$ \\
\hline Irradiation tool & $>10 \mathrm{MW}$ & $\sim 1 \mathrm{GeV}$ & $10 \mathrm{~mA}$ \\
\hline Muons - Neutrinos & $4 \mathrm{MW}$ & $2 \mathrm{GeV}$ & $2 \mathrm{~mA}$ \\
\hline
\end{tabular}

The linear accelerator is the only type of equipment with which such high intensities can be reached. Space charge effects set the limit at low energy and the RFQ (Radio Frequency Quadrupole) can accept up to $100 \mathrm{~mA}$ $\mathrm{cw}$ as experimentally demonstrated with LEDA (Low Energy Demonstration Accelerator) at Los Alamos.

\subsection{Reliability}

As far as reliability is concerned, the hybrid reactor application is by far the most demanding as it requires a very limited number of unscheduled beam interruptions, of the order of 100 per year at maximum provided the target and reactor designs are optimised for this. In comparison, the specification for the study of condensed matter by neutron scattering is relaxed by several hundreds. The present statistics for linacs in operation give about two orders of magnitude higher than the ADT specifications. It is evident that the equipment involved was not designed according to severe reliability criteria. More recently, high reliability levels have been demanded and achieved on synchrotron light rings of the third generation with 300 unscheduled interruptions per year. Means are available of doing substantially better and thus meeting the objective of one hundred. Efforts could also be pursued in parallel to achieve greater tolerance on the reactors and targets side.

\subsection{Multipurpose facility}

Although the ADT, TIT and RIB applications do not need any time structure and would naturally have specified $\mathrm{cw}$ beams, it was recognized that they can operate in a pulsed regime in the $10-100 \mathrm{~Hz}$ frequency range. It is then possible to meet the requirements with a single accelerator saving both driver construction and 
operation costs. Other important savings are also expected from common R\&D programs for the different applications, studies and designs of the spallation targets, infrastructures (buildings, power lines, utilities etc.), safety aspects...

A series of $50 \mathrm{~Hz}$ pulses can for example be distributed over the $20 \mathrm{~ms}$ period, each pulse duration being adjusted to satisfy the desired power of a given application. Assuming a $1.334 \mathrm{GeV}$ linac and $107 \mathrm{~mA}$ peak current, Fig. 1 shows a typical pulse sequence satisfying simultaneously most of the needs of Table 1 , resulting in a total beam power of $25 \mathrm{MW}$ with a duty cycle of $25 \%$. The interval between successive pulses is about $100 \mu$ s to operate fast switching magnets without beam loss.

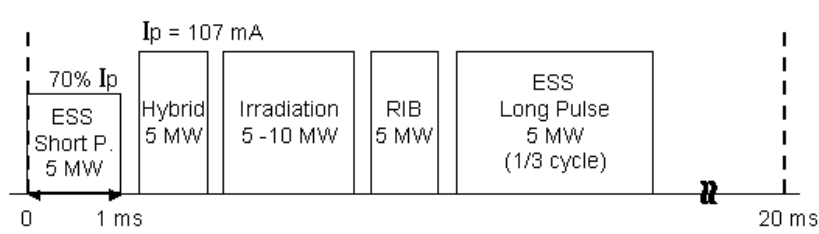

Figure 1: Typical pulse sequence.

All current pulses are protons, except the one attributed to the short pulse $(<1 \mu \mathrm{s})$ of the neutron source which consists of chopped $\mathrm{H}^{-}$beams for a proper injection into a compressor ring. Since the bunch charge is kept constant to maintain the same space-charge forces for all pulses, the average intensity of $\mathrm{H}^{-}$beams is then reduced with respect to proton beams by the chopper beam-on duty factor $(\sim 70 \%)$.

\subsection{Principles of the Linac design}

The accelerator includes the following components :

- the front end with one proton and two $\mathrm{H}^{-}$sources,

- the low-energy beam transport lines and RFQ accelerators with fast chopper systems and funneling lines for $\mathrm{H}^{-}$ions up to about $20 \mathrm{MeV}$,

- a succession of copper (DTL, SDTL, CCL) structures up to $185 \mathrm{MeV}$,

- the high energy superconducting linac to raise the final energy to $1.3 \mathrm{GeV}$ with high accelerating gradients,

- one or two accumulator rings to meet the short pulse spallation neutron source requirements,

- the high-energy transport lines and distribution systems used to direct the beam to the different targets.

In order to be excellent at the design performance in terms of reliability, availability and flexibility, the driver has to be robust and based on well-proven technologies. It must be pointed out that the same beam parameters are fixed for all pulses (peak current, space charge, focusing) A major concern in the design of the linac is the minimisation of beam losses, in order to avoid activation of the machine and irradiation of the environment. The main constraint is to maintain losses below the commonly agreed limit for hands-on maintenance of $1 \mathrm{~W} / \mathrm{m}$. A careful beam dynamics design is therefore needed to avoid the formation of a particle halo that would finally be lost in the linac or in the transfer lines. In order to minimize the risk of emittance growth, halo formation and energy exchange between transverse and longitudinal planes, the basic rules consist in varying smoothly the focusing parameters, in matching carefully the beam at the transitions between different structures and in avoiding the crossing of resonances.

\section{FRONT END \& LOW ENERGY PART}

The reference solution [6] of the low energy part was defined during a meeting held in Jülich in December 2000 and is shematically described in Fig. 2.

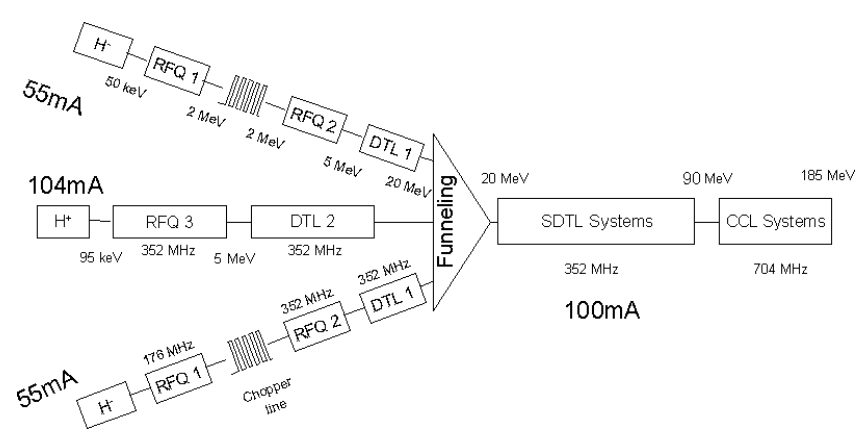

Figure 2: Reference solution of the low energy part.

The front end has to deliver $\mathrm{H}^{+}$and $\mathrm{H}^{-}$beams of about $100 \mathrm{~mA}$ peak current and the $\mathrm{H}^{-}$pulse has to be chopped at the ring revolution frequency $(1 \mathrm{MHz})$. As one single $\mathrm{H}^{-}$source is not able to fulfill the current requirement, two front ends are funnelled at $23 \mathrm{MeV}$. Each arm consists of a $60 \mathrm{~mA}$ ion source, a low energy beam transport, a $2 \mathrm{MeV}$ bunched beam transfer line between two RFQs for installing a fast chopping device and a DTL section. The $352 \mathrm{MHz}$ frequency is a good compromise for the low energy part, where the space charge effect is predominant, between large dimensions at lower frequency and better shunt impedance at higher frequency. Since the principle of the funnel implies a doubling of the bunch frequency, the first RFQ section is then at $176 \mathrm{MHz}$. However, the jump frequency of the cavities has been preferred in the second RFQ (perfectly suited for a beam bunching) before the funnel line, where smaller cavity dimensions are preferable. A $5 \mathrm{MeV}$ energy at the RFQ output allows for the use of electro-magnets instead of permanent magnets in the following $352 \mathrm{MHz}$ DTL structure and then for a fine tuning to avoid any halo development. The $20 \mathrm{MeV}$ energy at which funnelling takes place is a compromise between too high beam rigidity at higher energy and too high space-charge effects during beam transport at lower energy. The deflection is achieved by a special cavity, proposed by Y. Senichev [7]. For the proton beam, a single source can meet the beam requirement. Excellent avaibility and reliability of $100 \mathrm{~mA}$ ECR sources have been demonstrated for the LEDA and IPHI projects. The RFQ and DTL of the proton line have to operate with a higher duty cycle $(20 \%)$ than those of the $\mathrm{H}^{-}$line. After 
the funnel section a $352 \mathrm{MHz}$ Separarated-type Drift Tube Linac, which offers many advantages with respect to the classical DTL (higher shunt-impedance, fewer matching sections, less transitions,...) accelerates the beam to $90 \mathrm{MeV}$ and further acceleration to $185 \mathrm{MeV}$ is by a $704 \mathrm{MHz}$ Coupled Cavity Linac. The transition energy is determined by the crossing of the shunt impedance curves of both structures as a function of energy. Cost estimation showed that the cost variation of the CCL structure as a function of the field is very flat between 2 and $4 \mathrm{MV} / \mathrm{m}$.

\section{HIGH ENERGY PART}

The transition energy between NC and SC structures has been fixed at $185 \mathrm{MeV}$. Below $200 \mathrm{MeV}$, the energy gain per real-estate of SC cavities is lower than $2 \mathrm{MeV} / \mathrm{m}$, which can be obtained by warm Coupled Cavity Linac (CCL) structures. Besides, the design of SC cavities for beta values lower than 0.6 is complicated by stiffness and microphonics issues. For the present design, a $50 \mathrm{mT}$ peak magnetic field has been selected, a conservative factor of two lower than the surface field aimed at the TESLA project. Taking into account 3 fundamental constraints (maximum accelerating field, which depends on the cavity "beta"; longitudinal phase advance per cell below $90^{\circ}$ for stability reasons; beam power per cavity below the input coupler capability) the entire SRF linac architecture (in terms of number of cavity families, number of cells per cavity and number of cavities per cryomodule) was inferred from an optimisation of the linac length, which makes efficient use of the SC cavities [8]. Two cavity types have been selected, with a transition energy at $450 \mathrm{MeV}$. The total SRF linac length is lower than $300 \mathrm{~m}$, and a schematic layout is shown in Fig. 2, assuming realistic dimensions of cryostat and warm section for doublet focusing and diagnostics. One full 4-cavity module has been added at the linac end for redundancy purpose. The energy gain per cavity is shown in Fig. 3 as a function of the beam energy, with a synchronous phase set at $-25^{\circ}$. Assuming that two couplers are feeding each cavity, a maximum beam power of about $1 \mathrm{MW}$ per cavity was then chosen. The RF system is required to transfer RF power to the successive beam pulses over each $20 \mathrm{~ms}$ cycle.

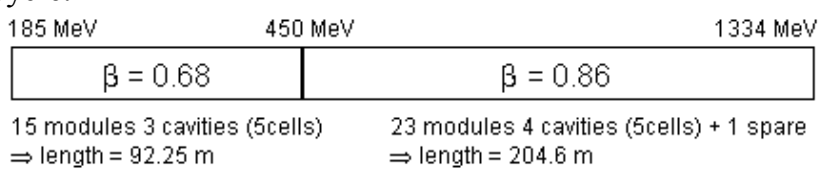

Figure 2: Schematic linac layout

As the RF power needed greatly varies, from $500 \mathrm{~kW}$ at the low-energy end to $1400 \mathrm{~kW}$ at the high-energy end, two klystron types are used (45 klystrons with $950 \mathrm{~kW}$ and 96 klystrons with $1400 \mathrm{~kW}$ saturation power). The "one klystron per cavity" scheme, where each cavity has its own feedback/feedforward RF control system, has been chosen because it provides the best RF stability for proton beams, the simplest operation procedure and the greatest flexibility in the event of a sudden RF failure.

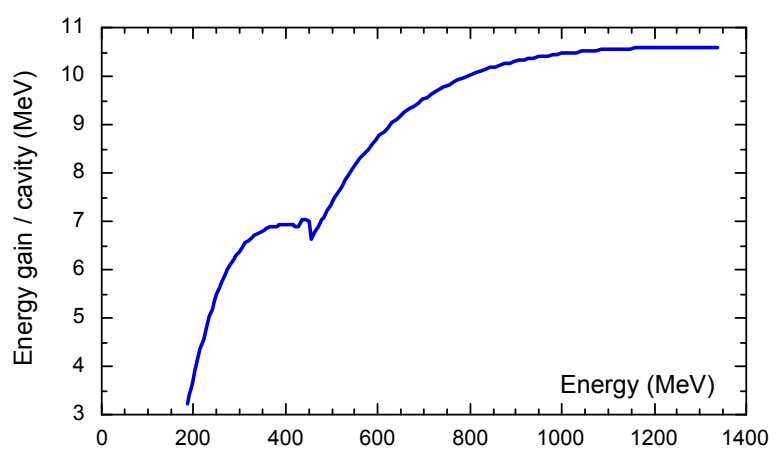

Figure 3: Energy gain per cavity along the linac.

Multiparticle simulations were carried out in the error free machine first with a matched input beam to check the overall architecture as well as beam matching, and second with a mismatched input beam to test the "robustness" of the linac design. Last, end-to-end simulations without errors [9] showed excellent beam qualities without significant beam tails (only $10^{-3}$ particles outside 3 times the rms size).

\section{REFERENCES}

[1] http://www.ess-europe.de

[2] http://www.ganil.fr/eurisol

[3] Proc. of the $3^{\text {rd }}$ Int. Conf. on Accelerator Driven Transmutation Technologies and Applications, June 1999, Praha, Czeck Republic.

[4] J.P. Deffain and J. Bergeron, AccApp'98, Gatlinburg, September 1998.

[5] B. Autin et al, CERN 2000-012, December 2000.

[6] R. Ferdinand et al, "Low Energy Part of the CONCERT High-Power Proton Linac", these proceedings.

[7] Y. Senichev, ESS note, ESS103-00-A, June 2000.

[8] A. Mosnier et al, "SC Proton Linac for the CONCERT multi-users Facility", these proceedings.

[9] N. Pichoff et al, "Beam Dynamics through the CONCERT Linac", these proceedings 